\title{
Coronavirus disease (COVID-19)
}

\section{Weekly Epidemiological Update}

Data as received by WHO from national authorities, as of 10 am CEST 30 August 2020

For the latest data and information on COVID-19, please see:

- WHO COVID-19 Dashboard

- Rolling updates on COVID-19

- WHO COVID-19 Weekly Operational Update

\section{Global epidemiological situation}

Over 1.8 million new COVID-19 cases and 38000 new deaths were reported to WHO in the week ending 30 August, a $1 \%$ increase in the number of cases and 3\% decrease in the number of deaths compared to the previous week (17 to 23 August) (Figure 1). A cumulative total of nearly 25 million cases and 800000 deaths have been reported since the start of the outbreak.

Figure 1: Number of COVID-19 cases and deaths reported weekly by WHO region, 30 December to 30 August 2020**

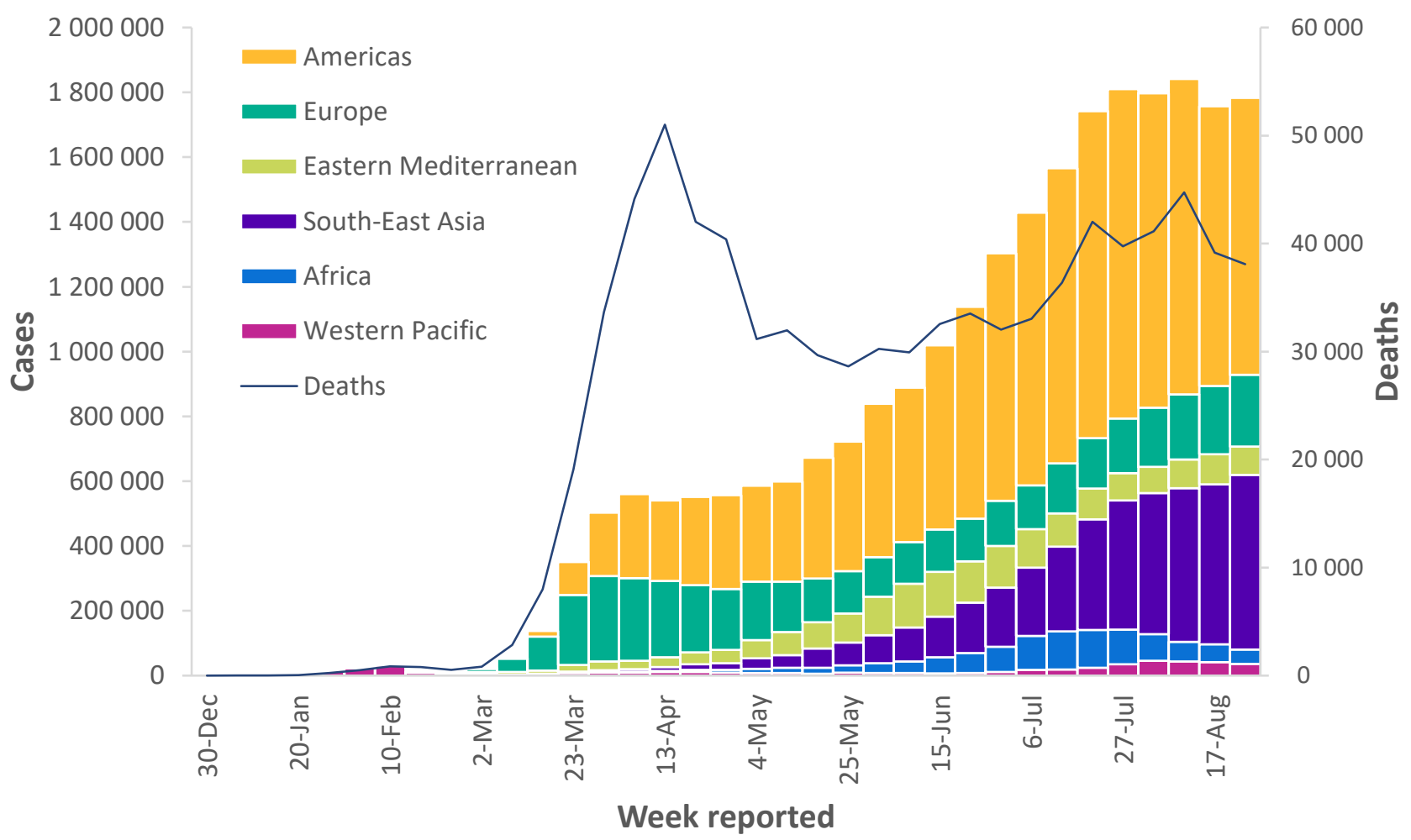

${ }^{* *}$ See data table and figure notes. 
Overall, the Region of the Americas continues to carry the highest burden of COVID-19 globally, accounting for nearly half of all new cases reported in the past seven days, although within the region there have been slight decreases in new cases and deaths in the past week. The WHO South East Asia Region showed the highest rise in new cases in the past week, with over 500,000 new cases reported. In the European Region, new cases and new deaths have continued to increase over the past seven days compared to the previous week. Along with the Region of the Americas, the percentage change in new cases in Africa, the Eastern Mediterranean, and Western Pacific Regions have all declined compared with last week.

Region specific information can be found below: African Region, Region of the Americas, Eastern Mediterranean Region, European Region, South-East Asia Region, Western-Pacific Region.

Table 1. Newly reported and cumulative COVID-19 confirmed cases and deaths, by WHO Region, data as of 30 August 2020**

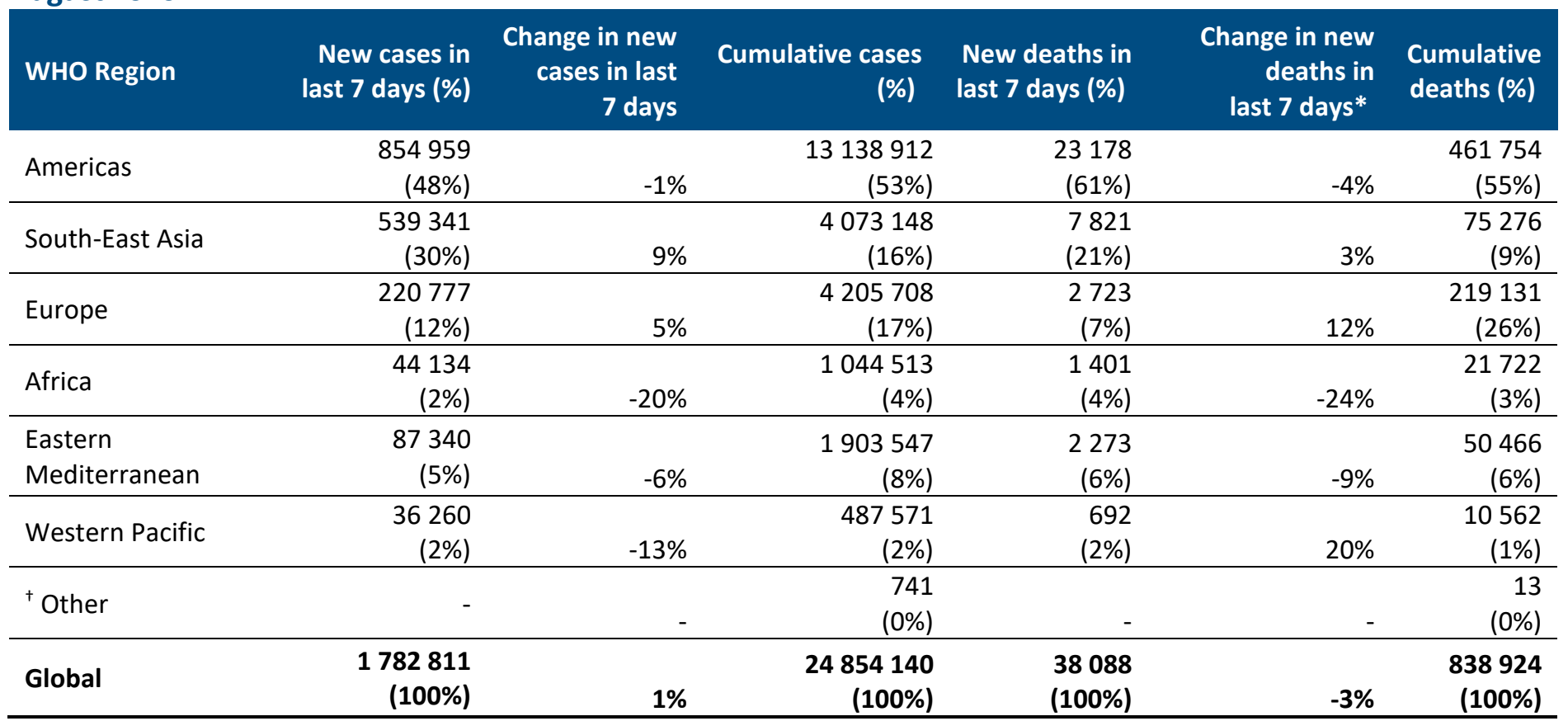

${ }^{*}$ Percent change in the number of newly confirmed cases/deaths in past seven days, compared to seven days prior.

${ }^{* *}$ See data, table and figure notes 
Figure 2. COVID-19 cases per 1 million population reported in the last seven days by countries,territories and areas, 24 August to 30 August $2020 * *$

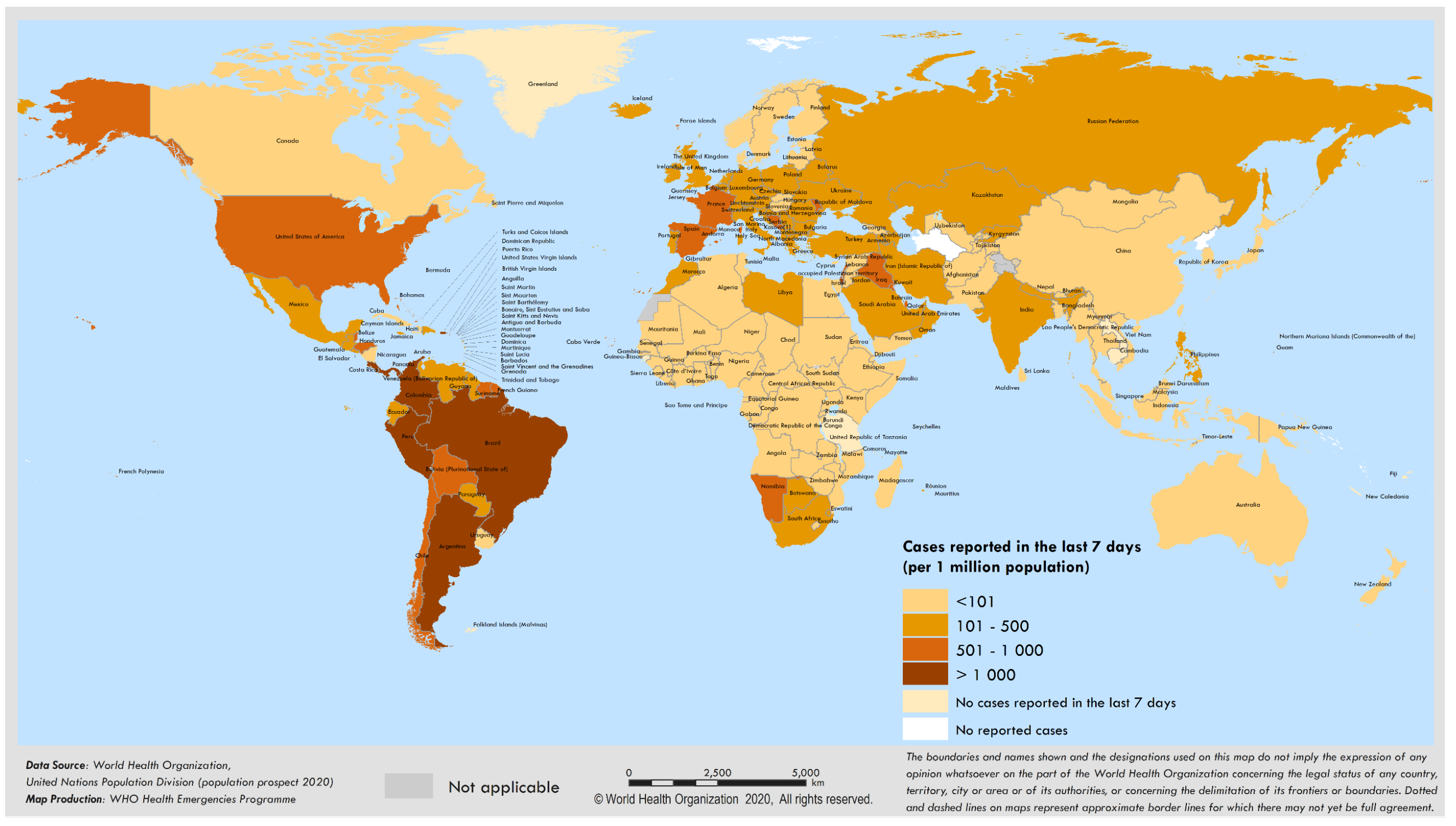

**See data, table and figure notes. 


\section{Situation by WHO Region}

\section{African Region}

Several countries in the Region that were previous COVID-19 hotspots have recorded a reduction in case incidence including Ghana, Kenya, Gabon and Madagascar. While the observed declining trends are encouraging, the figures should be interpreted cautiously as they may be affected by many factors, including the current testing capacity and strategy, and delays in reporting.

Cases in Ethiopia have continued to rise, reaching new highs this week (10 621 cases) with Addis Ababa remaining the worst affected region. In response, the Ethiopian government, with support from WHO, have launched the COVID-19 Enhanced Community Based Activities and Testing Campaign (ComBAT). ComBAT aims to reach out to over 60 million individuals in a variety of settings, conduct 200000 tests and treat 10000 cases.

At 622551 cases, South Africa has the fifth highest number of COVID-19 infections globally and the highest number in Africa. However, there has been a marked decline in new cases since they peaked on 25 July at 13944 cases, and the downward trend has continued this week with new cases falling by $34 \%$ (Figure 3). As a result, the government has eased public health and social measures while reminding the public to remain vigilant.

Figure 3: Number of COVID-19 cases and deaths reported weekly by the WHO African Region, data as of 30 August 2020**

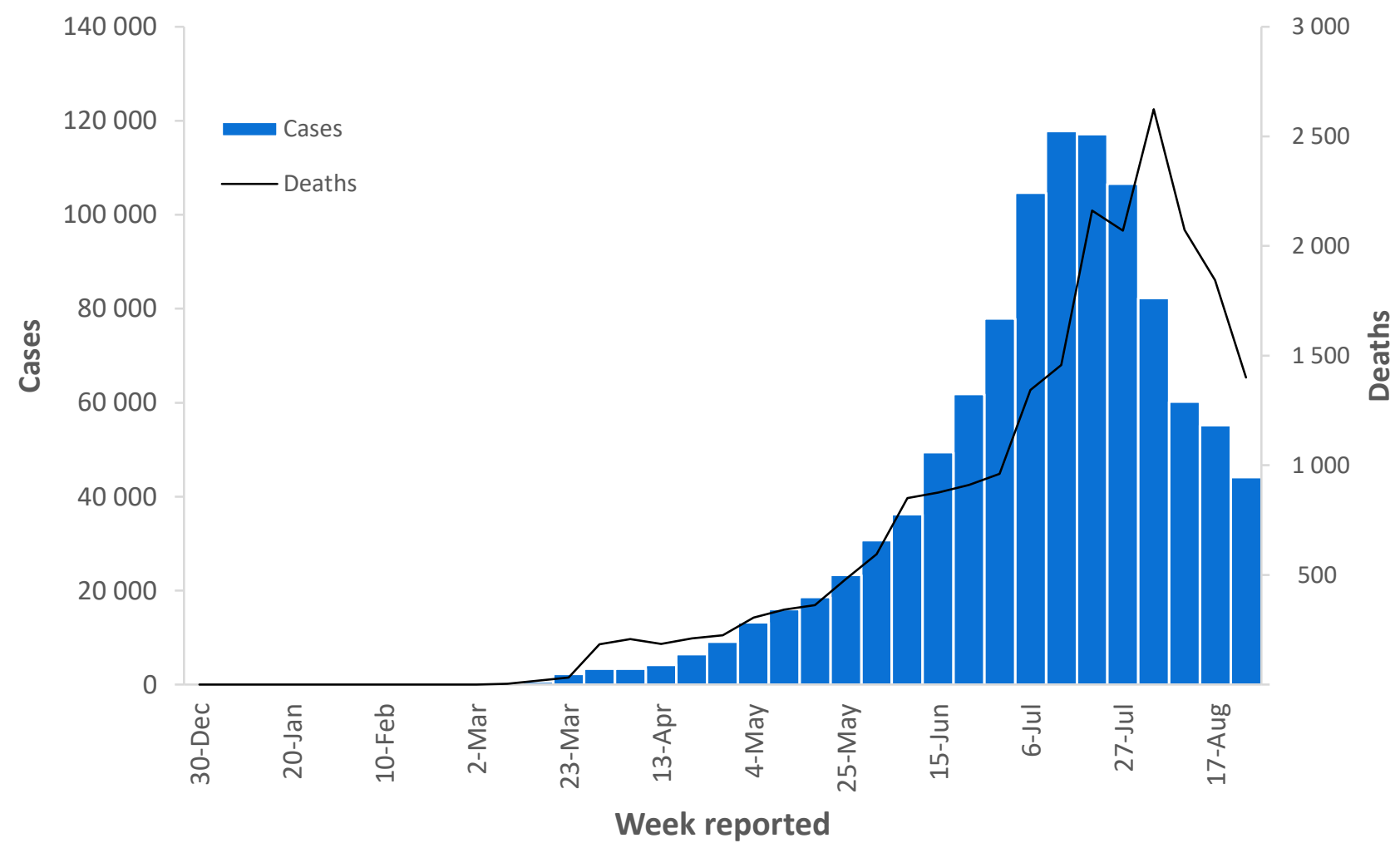




\section{Region of the Americas}

The Americas account for approximately $13 \%$ of the world's population, but over $50 \%$ of officially reported global cases and deaths. Currently, while the biggest drivers of the case counts in the region have observed a moderate decline or stabalisation of case incidence (Figure 4), increasing trends are now being seen in other parts of the region including Peru, Mexico, Colombia, and Argentina. Social gatherings could also have contributed to the increase in cases in the region. WHO recommends avoiding the 'three c's'-crowded places, close contact settings, and confined and enclosed settings. More advice from WHO on how to protect yourself and others from the spread COVID-19 can be found here.

The United States of America has the highest number of cases in the world - over 5.8 million cases, a quarter of the global total. However, the country has witnessed a decline in new cases from previous peaks in July of over 70000 cases per day to an average of 41000 new cases per day this past week. As school and university campuses have reopened, there have been news reports of students testing positive. WHO has published guidance for school-related public health measures in the context of COVID-19.

Although cases in Colombia have declined in the past week, they have been gradually increasing since June and continued caution is required. After five months of implementing public health and social measures, Colombia will begin easing the measures beginning 1 September.

Argentina has reported new peaks in the number of cases cases reported weekly and an upward trajectory in cases and deaths since June. Cases in Mexico declined by $6 \%$ in the past week and have declined gradually across the month of August. Mexico is implementing remote teaching and education for children to reduce transmission.

Figure 4: Number of COVID-19 cases and deaths reported weekly by Region of the Americas, data as of $\mathbf{3 0}$ August $2020^{* *}$

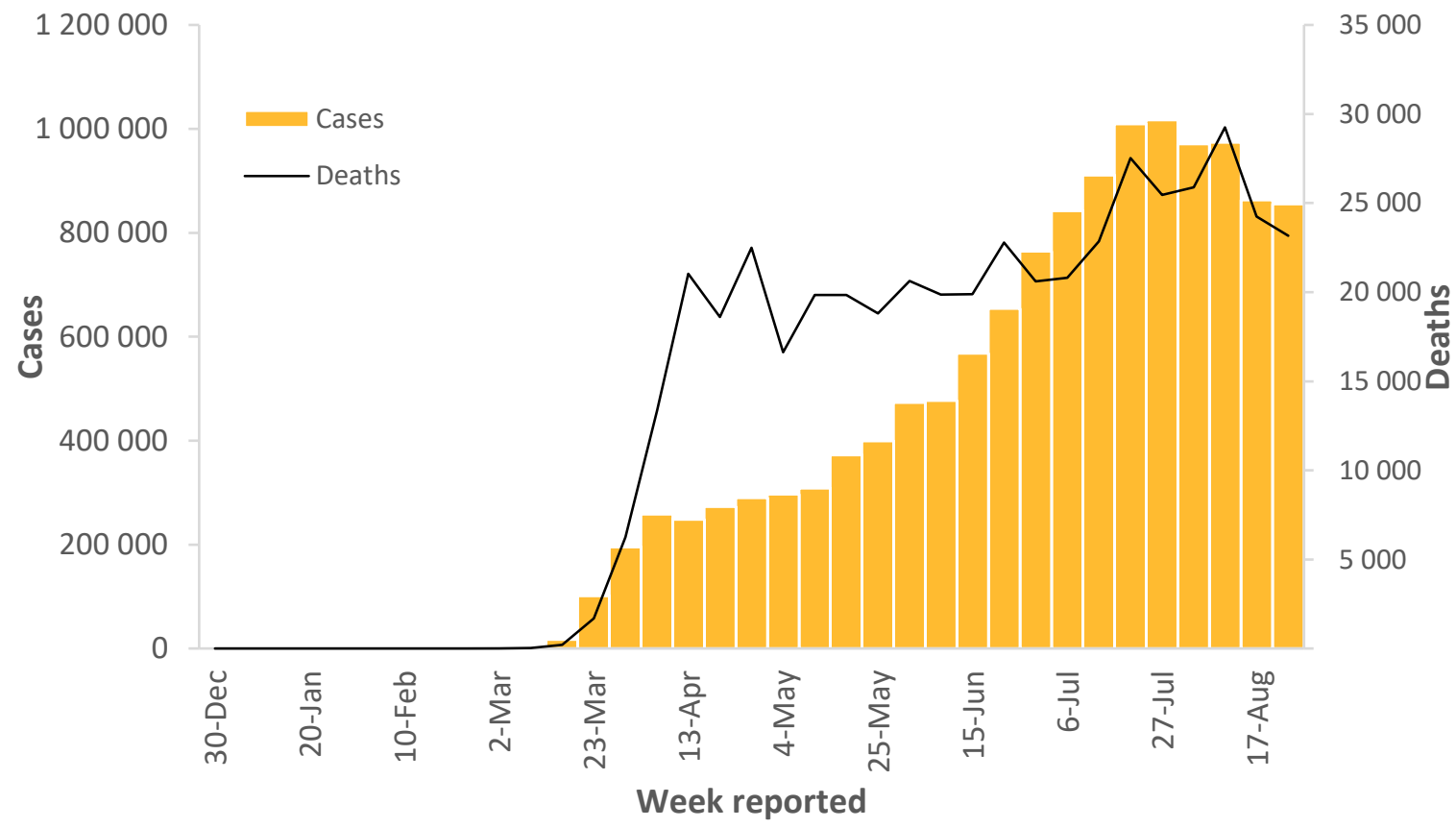




\section{Eastern Mediterranean Region}

The Eastern Mediterranean Region has observed a fluctuating incidence of new cases in recent weeks (Figure 5). The highest number of new cases have been seen in Iraq, Iran, Morocco, Saudi Arabia, and Kuwait. WHO, in partnership with the Iraqi Ministry of Health and its implementing partners, has concluded another round of a COVID-19 awareness-raising campaign that intensified community outreach efforts to educate people on measures to limit transmission of COVID-19. The campaign reached more than 5 million people in four densely populated and heavily affected areas in Basra, Wasit, Thi Qar and Missan. Campaign teams are moving to the Kurdistan region and will be targeting 9 heavily populated, high-risk areas in Sulaymaniyah province. Over the past week WHO has also delivered 6 ambulances to the Ministry of Health of the Kurdistan region and 22 tonnes of COVID-19 equipment.

In Pakistan, cases have fallen from over 5000 per day in mid-June to 2871 cases in the past week. As the public health and social measures are lifted, the public is encouraged to take precautions to ensure a resurgence does not occur particularly as they celebrate the first 10 days of the month of Muharram, which started on 21 August.

Figure 5: Number of COVID-19 cases and deaths reported weekly by Eastern Mediterranean Region, data as of 30 August $2020^{* *}$

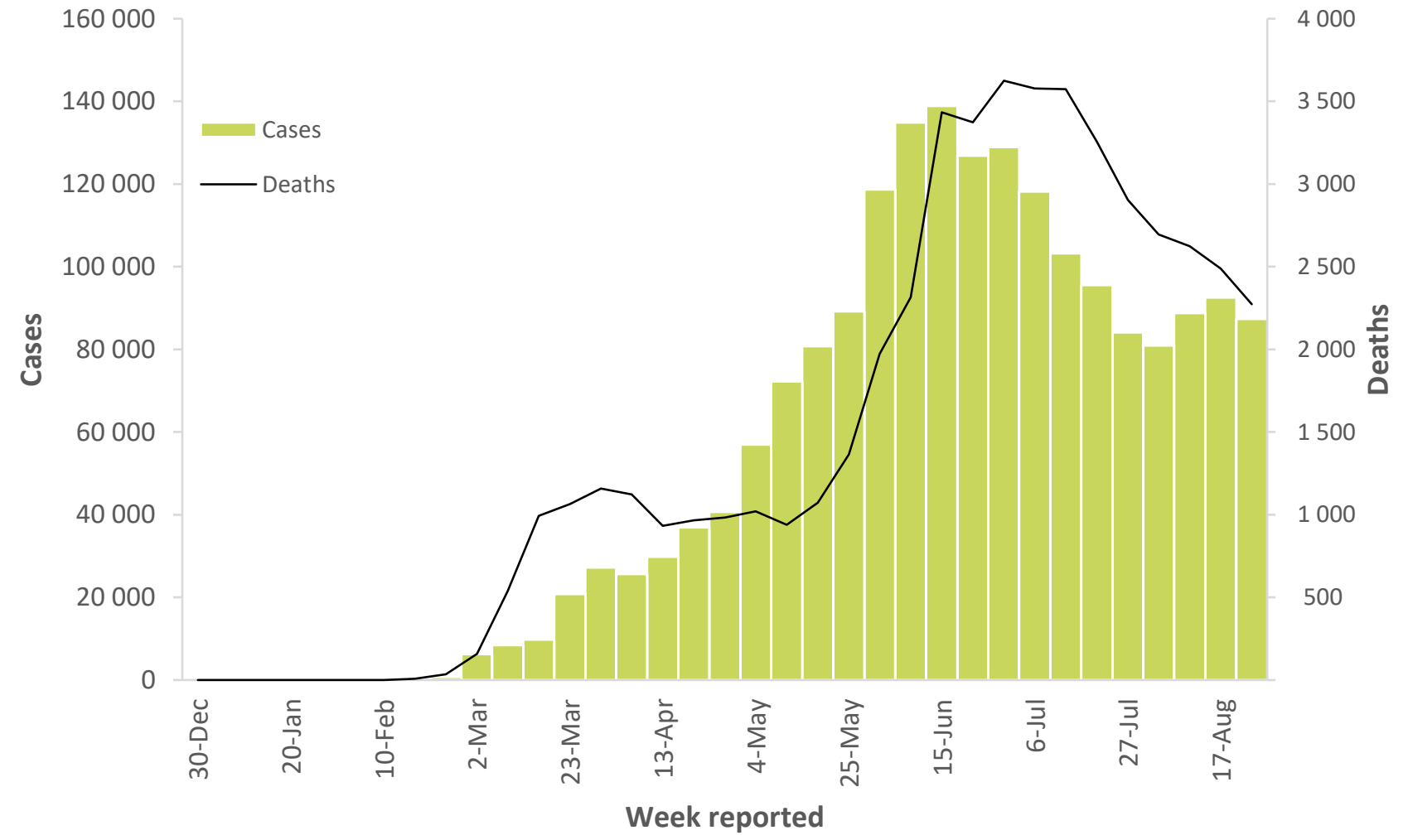

${ }^{* *}$ See data, table and figure notes 


\section{European Region}

Gradual increases continue to be observed in the European Region (Figure 6), with Spain, Russia, France, and Ukraine reporting the highest number of new cases this week. Spain has seen a resurgence since mid-July and cases are now reaching the peaks previously seen in March and April. July and August is the holiday season in Spain, and social interactions have likely contributed to the increased number of reported cases. Spain has deployed the military to support contact tracing activities.

France has also seen a growing number of cases since the end of July with cases rising from around 500 cases per day to approximately 4773 cases per day over the past week.

Cases in Italy have shown a marked increase in the past seven days, up by $85 \%$ overall compared to last week.

In the European Region many of those of who died of COVID-19 have been elderly people (as of this week $88 \%$ of all deaths were in persons aged 65 years and over). WHO has published comprehensive guidance on preventing and managing COVID-19 across long-term care services.

Figure 6: Number of COVID-19 cases and deaths reported weekly by European Region, data as of 30 August $2020^{* *}$

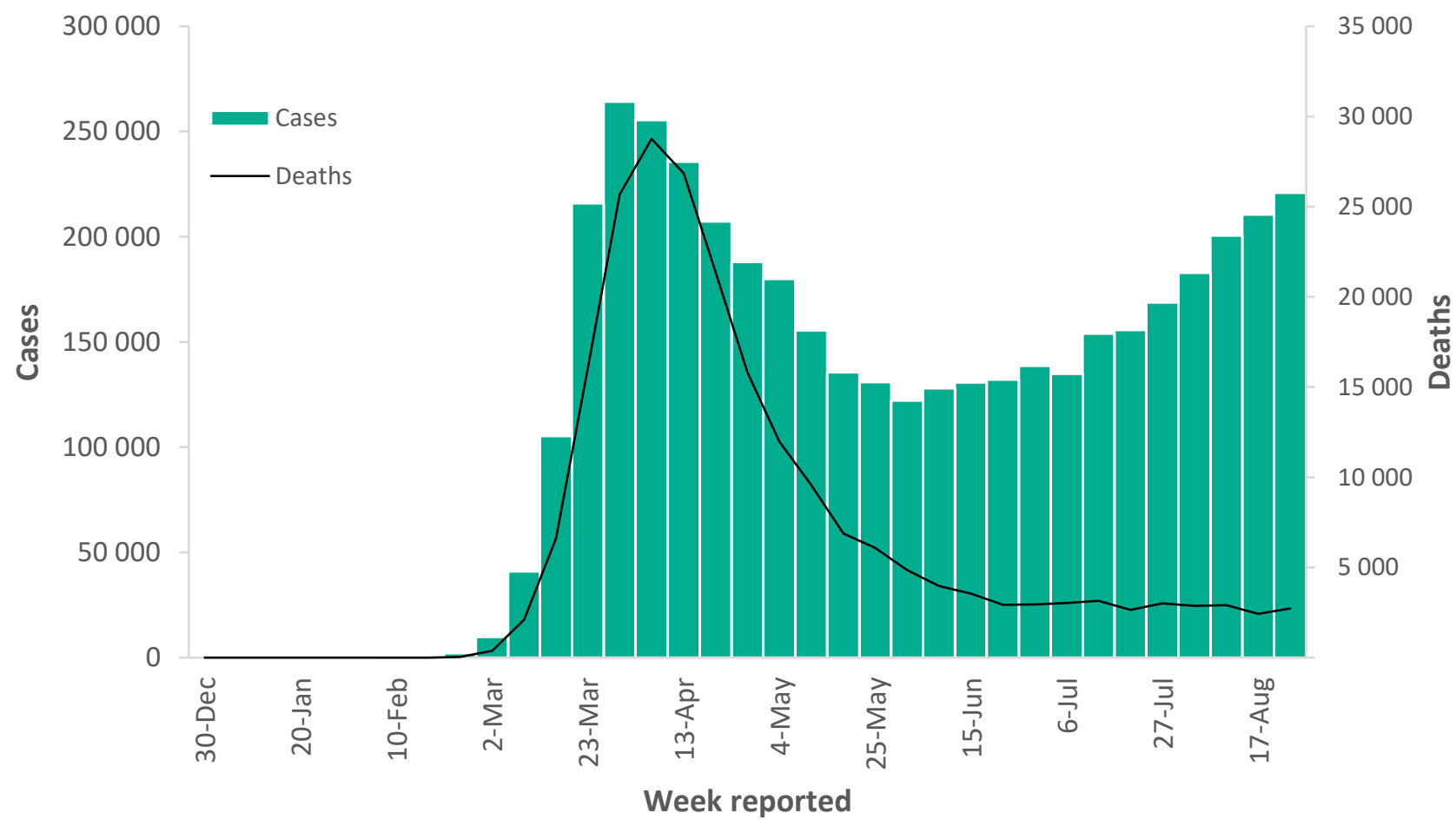

**See data, table and figure notes 


\section{South-East Asia Region}

South-East Asia has reported the largest week-on-week increase (Figure 7), largely due to increased case detections in India. India has reported nearly 500000 new cases in the past seven days, a 9\% increase compared to the previous seven days and the highest numbers of new cases globally. While these trends are concerning, the increase in cases should be seen against a substansive rise in testing in recent weeks.

In Indonesia, cases have been gradually increasing while there are also concerns about transmission among family members of school children as $40 \%$ of people aged 60 years and older in Indonesia live in three-generation households, meaning that they live with their children and grandchildren. WHO has published interim guidance on considerations for school-related public health measures in the context of COVID-19 and a joint document with UNICEF and IFRC on key messages and actions for COVID-19 prevention and control in schools. WHO has also convened a technical advisory group on schools.

Figure 7: Number of COVID-19 cases and deaths reported weekly by South-East Asia Region, data as of $\mathbf{3 0}$ August 2020**

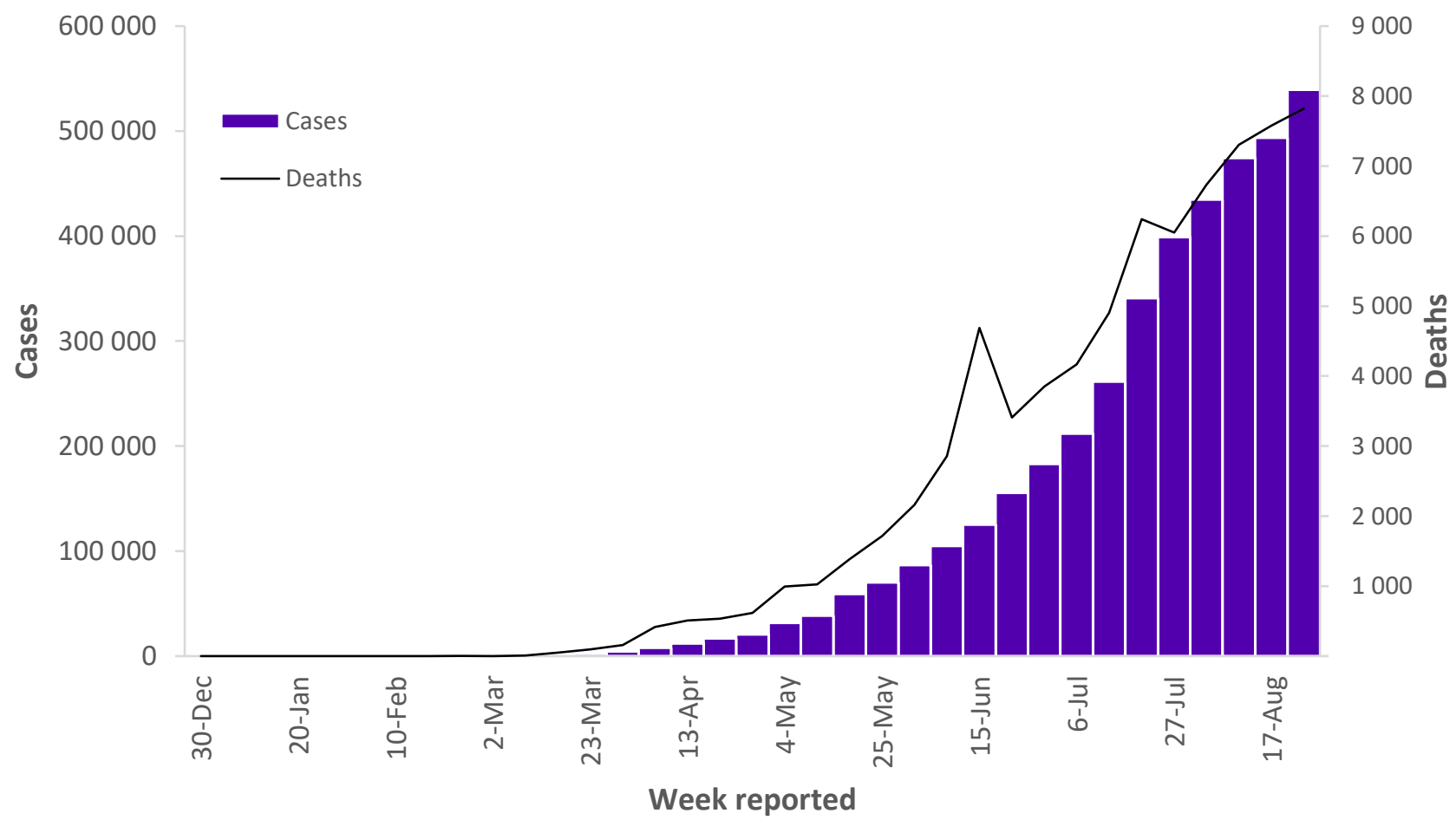

${ }^{* *}$ See data, table and figure notes 


\section{Western Pacific Region}

The Philippines and Japan are reporting the highest number of new cases in the region this week although cases in Japan have been decreasing since the end of June with a $22 \%$ decrease in overall cases reported this week compared to last week. In the Philippines, a large percentage of cases have been reported from the National Capital Region.

Figure 8: Number of COVID-19 cases and deaths reported weekly by Western Pacific Region, data as of $\mathbf{3 0}$ August 2020**

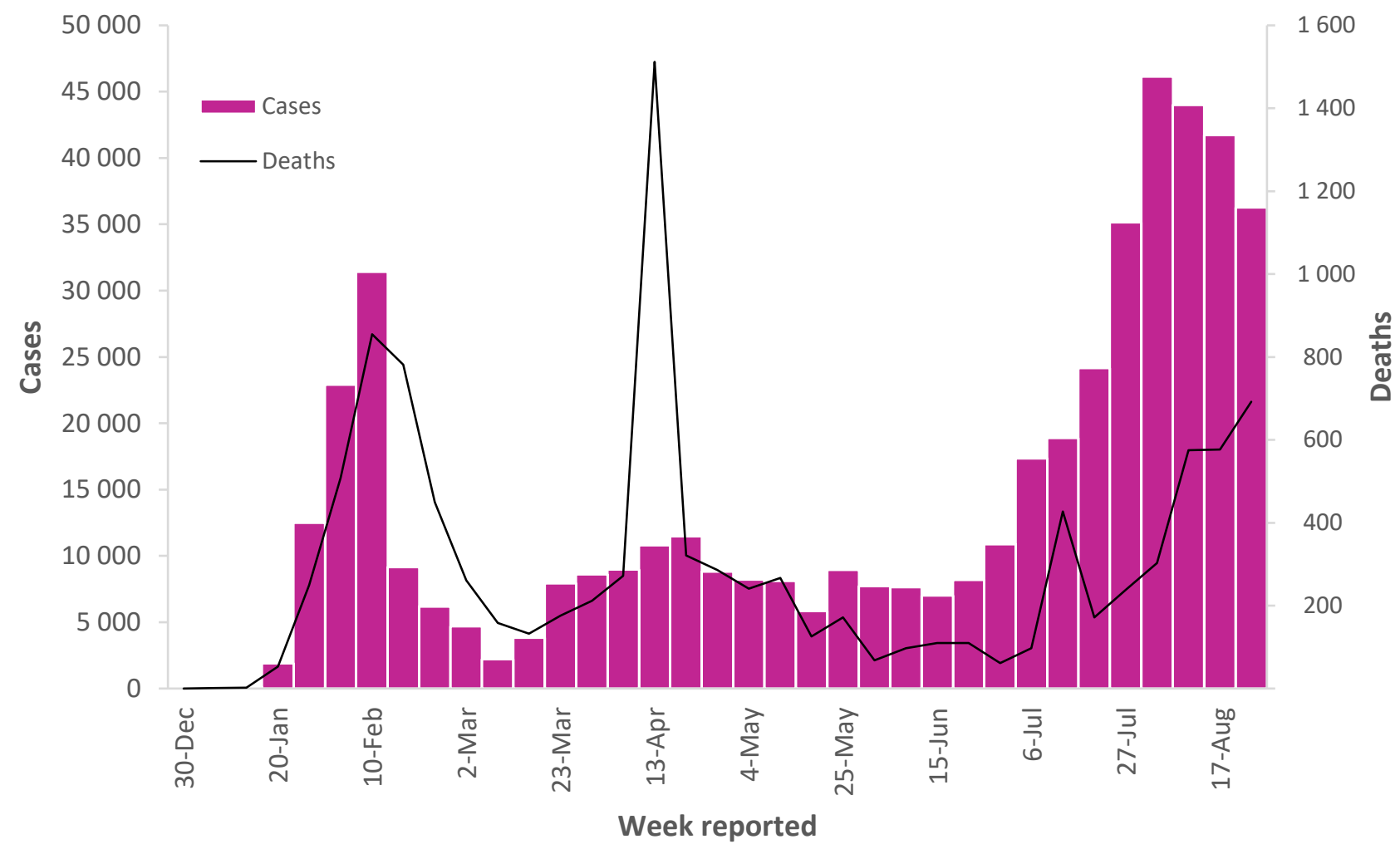

${ }^{* *}$ See data, table and figure notes 
Table 2. Number of COVID-19 confirmed cases and deaths reported in the last seven days by countries, territories and areas, African Region, data as of 30 August 2020**

\begin{tabular}{|c|c|c|c|c|c|c|c|}
\hline Reporting Country/Territory/Area & $\begin{array}{l}\text { New cases } \\
\text { in last } 7 \\
\text { days }\end{array}$ & $\begin{array}{l}\text { Cumulative } \\
\text { cases }\end{array}$ & $\begin{array}{l}\text { Cumulative cases } \\
\text { per } 1 \text { million } \\
\text { population }\end{array}$ & $\begin{array}{l}\text { New } \\
\text { deaths in } \\
\text { last } 7 \text { days }\end{array}$ & $\begin{array}{l}\text { Cumulative } \\
\text { deaths: }\end{array}$ & $\begin{array}{l}\text { Cumulative deaths } \\
\text { per } 1 \text { million } \\
\text { population }\end{array}$ & Transmission classification \\
\hline Africa & 44134 & 1044513 & 157 & 1401 & 21722 & 3 & \\
\hline South Africa & 15506 & 622551 & 10497 & 994 & 13981 & 236 & Community transmission \\
\hline Ethiopia & 10621 & 49654 & 432 & 108 & 770 & 7 & Community transmission \\
\hline Algeria & 2714 & 43782 & 998 & 67 & 1491 & 34 & Community transmission \\
\hline Nigeria & 1822 & 53727 & 261 & 14 & 1011 & 5 & Community transmission \\
\hline Kenya & 1676 & 33794 & 628 & 30 & 572 & 11 & Community transmission \\
\hline Namibia & 1578 & 7116 & 2801 & 23 & 69 & 27 & Community transmission \\
\hline Zambia & 1275 & 11902 & 647 & 7 & 284 & 15 & Community transmission \\
\hline Rwanda & 954 & 3843 & 297 & 5 & 16 & 1 & Clusters of cases \\
\hline Ghana & 624 & 43949 & 1414 & 9 & 270 & 9 & Community transmission \\
\hline Senegal & 606 & 13456 & 804 & 16 & 282 & 17 & Community transmission \\
\hline Uganda & 590 & 2756 & 60 & 8 & 28 & 1 & Clusters of cases \\
\hline Côte D'Ivoire & 519 & 17893 & 678 & 2 & 115 & 4 & Community transmission \\
\hline Madagascar & 514 & 14791 & 534 & 12 & 190 & 7 & Community transmission \\
\hline Zimbabwe & 513 & 6406 & 431 & 43 & 196 & 13 & Community transmission \\
\hline Mozambique & 456 & 3760 & 120 & 2 & 22 & 1 & Community transmission \\
\hline Angola & 417 & 2551 & 78 & 13 & 107 & 3 & Clusters of cases \\
\hline Cameroon & 380 & 19142 & 721 & 3 & 411 & 15 & Community transmission \\
\hline
\end{tabular}




\begin{tabular}{|c|c|c|c|c|c|c|c|}
\hline Gambia & 360 & 2797 & 1157 & 12 & 96 & 40 & Community transmission \\
\hline Botswana & 325 & 1633 & 694 & 3 & 6 & 3 & Clusters of cases \\
\hline Cabo Verde & 323 & 3778 & 6795 & 2 & 39 & 70 & Clusters of cases \\
\hline Eswatini & 321 & 4510 & 3887 & 8 & 91 & 78 & Community transmission \\
\hline Guinea & 284 & 9251 & 704 & 6 & 59 & 4 & Community transmission \\
\hline Democratic Republic of The Congo & 183 & 9993 & 112 & 4 & 255 & 3 & Community transmission \\
\hline Togo & 178 & 1390 & 168 & 0 & 27 & 3 & Community transmission \\
\hline Malawi & 146 & 5528 & 289 & 6 & 174 & 9 & Community transmission \\
\hline Congo & 129 & 3979 & 721 & 1 & 78 & 14 & Community transmission \\
\hline Mauritania & 118 & 7012 & 1508 & 0 & 158 & 34 & Community transmission \\
\hline Gabon & 117 & 8505 & 3821 & 0 & 53 & 24 & Community transmission \\
\hline Burkina Faso & 60 & 1357 & 65 & 0 & 55 & 3 & Community transmission \\
\hline Mali & 58 & 2757 & 136 & 1 & 126 & 6 & Community transmission \\
\hline Lesotho & 51 & 1066 & 498 & 1 & 31 & 14 & Clusters of cases \\
\hline Sierra Leone & 39 & 2019 & 253 & 1 & 70 & 9 & Community transmission \\
\hline Benin & 30 & 2145 & 177 & 1 & 40 & 3 & Community transmission \\
\hline Chad & 26 & 1008 & 61 & 1 & 77 & 5 & Community transmission \\
\hline Central African Republic & 21 & 4700 & 973 & 0 & 61 & 13 & Community transmission \\
\hline South Sudan & 20 & 2519 & 225 & 0 & 47 & 4 & Community transmission \\
\hline Liberia & 18 & 1303 & 258 & 0 & 82 & 16 & Community transmission \\
\hline Burundi & 15 & 445 & 37 & 0 & 1 & $<1$ & Clusters of cases \\
\hline Equatorial Guinea & 15 & 4941 & 3522 & 0 & 83 & 59 & Community transmission \\
\hline Eritrea & 12 & 318 & 90 & 0 & 0 & $<1$ & Sporadic cases \\
\hline Comoros & 6 & 423 & 486 & 0 & 7 & 8 & Community transmission \\
\hline
\end{tabular}




\begin{tabular}{|c|c|c|c|c|c|c|c|}
\hline Seychelles & 4 & 131 & 1332 & 0 & 0 & $<1$ & Sporadic cases \\
\hline Niger & 3 & 1175 & 49 & 0 & 69 & 3 & Clusters of cases \\
\hline Sao Tome and Principe & 3 & 895 & 4084 & 0 & 15 & 68 & Clusters of cases \\
\hline Guinea-Bissau & 0 & 2149 & 1092 & 0 & 33 & 17 & Community transmission \\
\hline Mauritius & 0 & 346 & 272 & 0 & 10 & 8 & Sporadic cases \\
\hline United Republic of Tanzania & 0 & 509 & 9 & 0 & 21 & $<1$ & Community transmission \\
\hline \multicolumn{8}{|l|}{ Territoriesii } \\
\hline Réunion & 440 & 1557 & 1739 & -3 & 3 & 3 & Clusters of cases \\
\hline Mayotte & 64 & 3301 & 12100 & 1 & 40 & 147 & Clusters of cases \\
\hline Americas & 854959 & 13138912 & 1950 & 23178 & 461754 & 69 & \\
\hline United States of America & 288304 & 5855521 & 17690 & 6443 & 180689 & 546 & Community transmission \\
\hline Brazil & 272473 & 3804803 & 17900 & 6146 & 119504 & 562 & Community transmission \\
\hline Colombia & 68382 & 590520 & 11605 & 2199 & 18767 & 369 & Community transmission \\
\hline Argentina & 62966 & 392009 & 8674 & 1510 & 8305 & 184 & Community transmission \\
\hline Peru & 53894 & 629961 & 19106 & 1226 & 28471 & 863 & Community transmission \\
\hline Mexico & 36004 & 585738 & 4543 & 3536 & 63146 & 490 & Community transmission \\
\hline Chile & 12301 & 408009 & 21344 & 389 & 11181 & 585 & Community transmission \\
\hline Bolivia (Plurinational State of) & 6974 & 114409 & 9801 & 480 & 4846 & 415 & Community transmission \\
\hline Costa Rica & 6351 & 38485 & 7555 & 67 & 407 & 80 & Community transmission \\
\hline Panama & 6232 & 90624 & 21003 & 107 & 1966 & 456 & Community transmission \\
\hline Guatemala & 5823 & 73679 & 4113 & 148 & 2728 & 152 & Community transmission \\
\hline Ecuador & 5817 & 112906 & 6399 & 260 & 6537 & 371 & Community transmission \\
\hline
\end{tabular}




\begin{tabular}{|c|c|c|c|c|c|c|c|}
\hline Venezuela (Bolivarian Republic of) & 5691 & 43879 & 1543 & 41 & 358 & 13 & Community transmission \\
\hline Honduras & 5429 & 58810 & 5938 & 195 & 1827 & 184 & Community transmission \\
\hline Paraguay & 3338 & 15874 & 2226 & 98 & 280 & 39 & Community transmission \\
\hline Dominican Republic & 3165 & 93726 & 8640 & 119 & 1673 & 154 & Community transmission \\
\hline Canada & 2986 & 127358 & 3374 & 44 & 9108 & 241 & Community transmission \\
\hline El Salvador & 1117 & 25537 & 3937 & 54 & 708 & 109 & Community transmission \\
\hline Trinidad and Tobago & 678 & 1577 & 1127 & 6 & 19 & 14 & Community transmission \\
\hline Jamaica & 665 & 2011 & 679 & 3 & 19 & 6 & Clusters of cases \\
\hline Suriname & 388 & 3848 & 6559 & 10 & 66 & 113 & Community transmission \\
\hline Bahamas & 354 & 2057 & 5231 & 13 & 40 & 102 & Clusters of cases \\
\hline Cuba & 308 & 3925 & 347 & 5 & 94 & 8 & Clusters of cases \\
\hline Guyana & 299 & 1180 & 1500 & 5 & 35 & 44 & Clusters of cases \\
\hline Belize & 222 & 870 & 2188 & 7 & 12 & 30 & Community transmission \\
\hline Haiti & 124 & 8174 & 717 & 5 & 201 & 18 & Community transmission \\
\hline Nicaragua & 119 & 3659 & 552 & 4 & 137 & 21 & Community transmission \\
\hline Uruguay & 40 & 1556 & 448 & 1 & 43 & 12 & Clusters of cases \\
\hline Barbados & 9 & 166 & 578 & 0 & 7 & 24 & Clusters of cases \\
\hline Saint Vincent and the Grenadines & 2 & 60 & 541 & 0 & 0 & $<1$ & Sporadic cases \\
\hline Dominica & 1 & 20 & 278 & 0 & 0 & $<1$ & Clusters of cases \\
\hline Antigua and Barbuda & 0 & 94 & 960 & 0 & 3 & 31 & Clusters of cases \\
\hline Grenada & 0 & 24 & 213 & 0 & 0 & $<1$ & Clusters of cases \\
\hline Saint Kitts and Nevis & 0 & 17 & 320 & 0 & 0 & $<1$ & No cases \\
\hline Saint Lucia & 0 & 26 & 142 & 0 & 0 & $<1$ & Sporadic cases \\
\hline
\end{tabular}




\begin{tabular}{|c|c|c|c|c|c|c|c|}
\hline Puerto Rico & 2973 & 32550 & 11378 & 47 & 428 & 150 & Community transmission \\
\hline Aruba & 461 & 1848 & 17309 & 2 & 8 & 75 & Community transmission \\
\hline French Guiana & 279 & 9076 & 30387 & 3 & 58 & 194 & Community transmission \\
\hline United States Virgin Islands & 186 & 1118 & 10706 & 4 & 14 & 134 & Community transmission \\
\hline Guadeloupe & 164 & 935 & 2337 & 0 & 15 & 37 & Clusters of cases \\
\hline Turks and Caicos Islands & 143 & 490 & 12656 & 1 & 3 & 77 & Clusters of cases \\
\hline Martinique & 132 & 596 & 1588 & 0 & 16 & 43 & Clusters of cases \\
\hline Sint Maarten & 91 & 444 & 10354 & 0 & 17 & 396 & Community transmission \\
\hline Saint Martin & 37 & 213 & 5510 & 0 & 5 & 129 & Sporadic cases \\
\hline Curaçao & 18 & 55 & 335 & 0 & 1 & 6 & Sporadic cases \\
\hline British Virgin Islands & 14 & 35 & 1158 & 0 & 1 & 33 & Sporadic cases \\
\hline Bonaire, Sint Eustatius and Saba & 2 & 15 & 572 & 0 & 0 & $<1$ & Sporadic cases \\
\hline Saint Barthélemy & 2 & 18 & 1821 & 0 & 0 & $<1$ & Sporadic cases \\
\hline Bermuda & 1 & 168 & 2698 & 0 & 9 & 145 & Sporadic cases \\
\hline Anguilla & 0 & 3 & 200 & 0 & 0 & $<1$ & No cases \\
\hline Cayman Islands & 0 & 205 & 3119 & 0 & 1 & 15 & Sporadic cases \\
\hline Falkland Islands (Malvinas) & 0 & 13 & 3732 & 0 & 0 & $<1$ & No cases \\
\hline Montserrat & 0 & 13 & 2601 & 0 & 1 & 200 & No cases \\
\hline Saint Pierre and Miquelon & 0 & 5 & 863 & 0 & 0 & $<1$ & Sporadic cases \\
\hline Eastern Mediterranean & 87340 & 1903547 & 271 & 2273 & 50466 & 7 & \\
\hline Iraq & 26396 & 227446 & 5655 & 538 & 6891 & 171 & Community transmission \\
\hline Iran (Islamic Republic of) & 15024 & 371816 & 4427 & 857 & 21359 & 254 & Community transmission \\
\hline Morocco & 9244 & 60056 & 1627 & 220 & 1078 & 29 & Clusters of cases \\
\hline
\end{tabular}




\begin{tabular}{|c|c|c|c|c|c|c|c|}
\hline Saudi Arabia & 7541 & 313911 & 9017 & 221 & 3840 & 110 & Clusters of cases \\
\hline Kuwait & 4267 & 84224 & 19722 & 15 & 528 & 124 & Clusters of cases \\
\hline Lebanon & 4084 & 16275 & 2384 & 34 & 155 & 23 & Community transmission \\
\hline Pakistan & 2871 & 295636 & 1338 & 53 & 6288 & 28 & Clusters of cases \\
\hline United Arab Emirates & 2711 & 69328 & 7010 & 7 & 379 & 38 & Community transmission \\
\hline Libya & 2521 & 12958 & 1886 & 43 & 231 & 34 & Clusters of cases \\
\hline Bahrain & 2353 & 51391 & 30202 & 6 & 189 & 111 & Clusters of cases \\
\hline Qatar & 1642 & 118407 & 41099 & 3 & 196 & 68 & Community transmission \\
\hline Egypt & 1260 & 98497 & 963 & 133 & 5376 & 53 & Clusters of cases \\
\hline Oman & 1236 & 85005 & 16646 & 41 & 650 & 127 & Community transmission \\
\hline Tunisia & 834 & 3572 & 302 & 7 & 75 & 6 & Clusters of cases \\
\hline Sudan & 507 & 13189 & 301 & 8 & 823 & 19 & Community transmission \\
\hline Syrian Arab Republic & 485 & 2628 & 150 & 21 & 106 & 6 & Community transmission \\
\hline Jordan & 317 & 1893 & 186 & 4 & 15 & 1 & Clusters of cases \\
\hline Afghanistan & 144 & 38143 & 980 & 15 & 1402 & 36 & Clusters of cases \\
\hline Somalia & 41 & 3310 & 208 & 4 & 97 & 6 & Sporadic cases \\
\hline Yemen & 39 & 1950 & 65 & 17 & 564 & 19 & Community transmission \\
\hline Djibouti & 3 & 5385 & 5450 & 0 & 60 & 61 & Sporadic cases \\
\hline \multicolumn{8}{|l|}{ Territoriesii } \\
\hline Occupied Palestinian territory & 3820 & 28527 & 5592 & 26 & 164 & 32 & Clusters of cases \\
\hline Europe & 220777 & 4205708 & 615 & 2723 & 219131 & 32 & 0 \\
\hline Spain & 37308 & 439286 & 9396 & 132 & 29011 & 620 & Clusters of cases \\
\hline Russian Federation & 33577 & 990326 & 6786 & 710 & 17093 & 117 & Clusters of cases \\
\hline France & 33410 & 256829 & 3935 & 89 & 30465 & 467 & Community transmission \\
\hline Ukraine & 14116 & 119074 & 2723 & 256 & 2527 & 58 & Community transmission \\
\hline
\end{tabular}




\begin{tabular}{|c|c|c|c|c|c|c|c|}
\hline Israel & 11036 & 110863 & 12808 & 87 & 885 & 102 & Community transmission \\
\hline Turkey & 10032 & 267064 & 3167 & 182 & 6284 & 75 & Community transmission \\
\hline Germany & 8907 & 241771 & 2886 & 26 & 9295 & 111 & Clusters of cases \\
\hline Italy & 8717 & 266853 & 4414 & 43 & 35473 & 587 & Clusters of cases \\
\hline Romania & 8289 & 85833 & 4462 & 306 & 3539 & 184 & Community transmission \\
\hline The United Kingdom & 8151 & 332756 & 4902 & 75 & 41498 & 611 & Community transmission \\
\hline Poland & 5058 & 66239 & 1750 & 81 & 2032 & 54 & Community transmission \\
\hline Netherlands & 3529 & 69563 & 4060 & 24 & 6215 & 363 & Community transmission \\
\hline Kazakhstan & 3470 & 130673 & 6959 & 148 & 1781 & 95 & Clusters of cases \\
\hline Republic of Moldova & 3332 & 36404 & 9024 & 55 & 990 & 245 & Community transmission \\
\hline Uzbekistan & 2605 & 41303 & 1234 & 42 & 311 & 9 & Clusters of cases \\
\hline Belgium & 2329 & 84505 & 7291 & 25 & 9891 & 853 & Community transmission \\
\hline Czechia & 2304 & 24094 & 2250 & 10 & 421 & 39 & Clusters of cases \\
\hline Switzerland & 2089 & 41615 & 4808 & 5 & 1724 & 199 & Community transmission \\
\hline Portugal & 1996 & 57448 & 5634 & 24 & 1818 & 178 & Clusters of cases \\
\hline Croatia & 1961 & 9861 & 2402 & 13 & 183 & 45 & Community transmission \\
\hline Austria & 1838 & 26937 & 2991 & 1 & 733 & 81 & Community transmission \\
\hline Bosnia and Herzegovina & 1834 & 19546 & 5958 & 62 & 588 & 179 & Community transmission \\
\hline Greece & 1596 & 9977 & 957 & 20 & 260 & 25 & Clusters of cases \\
\hline Belarus & 1238 & 71523 & 7569 & 34 & 671 & 71 & Community transmission \\
\hline Azerbaijan & 1069 & 36174 & 3568 & 14 & 529 & 52 & Clusters of cases \\
\hline Bulgaria & 1033 & 16164 & 2326 & 66 & 605 & 87 & Clusters of cases \\
\hline Albania & 1004 & 9279 & 3224 & 30 & 275 & 96 & Clusters of cases \\
\hline
\end{tabular}




\begin{tabular}{|c|c|c|c|c|c|c|c|}
\hline North Macedonia & 985 & 14293 & 6860 & 39 & 596 & 286 & Clusters of cases \\
\hline Armenia & 958 & 43750 & 14764 & 25 & 877 & 296 & Community transmission \\
\hline Sweden & 857 & 83958 & 8313 & 2 & 5821 & 576 & Community transmission \\
\hline Ireland & 812 & 28720 & 5816 & 0 & 1777 & 360 & Clusters of cases \\
\hline Kyrgyzstan & 797 & 43820 & 6717 & 3 & 1059 & 162 & Clusters of cases \\
\hline Serbia & 734 & 31282 & 4492 & 15 & 710 & 102 & Community transmission \\
\hline Denmark & 573 & 16700 & 2883 & 3 & 624 & 108 & Community transmission \\
\hline Hungary & 536 & 5669 & 587 & 3 & 614 & 64 & Community transmission \\
\hline Slovakia & 526 & 3842 & 704 & 0 & 33 & 6 & Clusters of cases \\
\hline Montenegro & 421 & 4777 & 7606 & 12 & 96 & 153 & Clusters of cases \\
\hline Norway & 346 & 10543 & 1945 & 0 & 264 & 49 & Clusters of cases \\
\hline Malta & 270 & 1847 & 4183 & 0 & 10 & 23 & Sporadic cases \\
\hline Lithuania & 245 & 2839 & 1043 & 2 & 86 & 32 & Community transmission \\
\hline Tajikistan & 239 & 8516 & 893 & 2 & 68 & 7 & Pending \\
\hline Slovenia & 217 & 2834 & 1363 & 1 & 128 & 62 & Clusters of cases \\
\hline Finland & 178 & 8049 & 1453 & 1 & 335 & 60 & Community transmission \\
\hline Estonia & 98 & 2363 & 1781 & 1 & 64 & 48 & Clusters of cases \\
\hline Andorra & 77 & 1124 & 14547 & 0 & 53 & 686 & Community transmission \\
\hline Cyprus & 66 & 1483 & 1228 & 0 & 21 & 17 & Clusters of cases \\
\hline Georgia & 58 & 1469 & 368 & 2 & 19 & 5 & Community transmission \\
\hline Iceland & 42 & 2100 & 6154 & 0 & 10 & 29 & Community transmission \\
\hline Latvia & 42 & 1375 & 729 & 1 & 34 & 18 & Clusters of cases \\
\hline Monaco & 22 & 130 & 3313 & 0 & 1 & 25 & Sporadic cases \\
\hline San Marino & 7 & 733 & 21598 & 0 & 42 & 1238 & Community transmission \\
\hline Liechtenstein & 6 & 107 & 2806 & 0 & 1 & 26 & Sporadic cases \\
\hline
\end{tabular}




\begin{tabular}{|c|c|c|c|c|c|c|c|}
\hline Holy See & 0 & 12 & 14833 & 0 & 0 & $<1$ & Sporadic cases \\
\hline Luxembourg & -1124 & 6580 & 10512 & 0 & 124 & 198 & Community transmission \\
\hline \multicolumn{8}{|l|}{ Territoriesii } \\
\hline Kosovo[1] & 878 & 13172 & 7080 & 51 & 498 & 268 & Community transmission \\
\hline Gibraltar & 45 & 274 & 8133 & 0 & 0 & $<1$ & Clusters of cases \\
\hline Faroe Islands & 27 & 411 & 8411 & 0 & 0 & $<1$ & Sporadic cases \\
\hline Jersey & 11 & 374 & 3438 & 0 & 32 & 294 & Community transmission \\
\hline Greenland & 0 & 14 & 247 & 0 & 0 & $<1$ & No cases \\
\hline Guernsey & 0 & 252 & 3988 & 0 & 13 & 206 & Community transmission \\
\hline Isle of Man & 0 & 336 & 3951 & 0 & 24 & 282 & No cases \\
\hline South-East Asia & 539341 & 4073148 & 707 & 7821 & 75276 & 13 & 0 \\
\hline India & 497793 & 3542733 & 2567 & 6792 & 63498 & 46 & Clusters of cases \\
\hline Indonesia & 17697 & 169195 & 619 & 667 & 7261 & 27 & Community transmission \\
\hline Bangladesh & 16300 & 308925 & 1876 & 299 & 4206 & 26 & Community transmission \\
\hline Nepal & 6223 & 37340 & 1282 & 61 & 207 & 7 & Clusters of cases \\
\hline Maldives & 918 & 7578 & 14019 & 2 & 28 & 52 & Clusters of cases \\
\hline Myanmar & 305 & 749 & 14 & 0 & 6 & $<1$ & Clusters of cases \\
\hline Sri Lanka & 48 & 2995 & 140 & 0 & 12 & 1 & Clusters of cases \\
\hline Bhutan & 40 & 195 & 253 & 0 & 0 & $<1$ & Sporadic cases \\
\hline Thailand & 16 & 3411 & 49 & 0 & 58 & 1 & Clusters of cases \\
\hline Timor-Leste & 1 & 27 & 20 & 0 & 0 & $<1$ & No cases \\
\hline Western Pacific & 36260 & 487571 & 84 & 692 & 10562 & 2 & 0 \\
\hline Philippines & 25882 & 213131 & 1945 & 453 & 3419 & 31 & Community transmission \\
\hline
\end{tabular}




\begin{tabular}{|c|c|c|c|c|c|c|c|}
\hline Japan & 5517 & 67264 & 532 & 88 & 1264 & 10 & Clusters of cases \\
\hline Republic of Korea & 2300 & 19699 & 384 & 14 & 323 & 6 & Clusters of cases \\
\hline Australia & 945 & 25547 & 1002 & 115 & 600 & 24 & Clusters of cases \\
\hline Singapore & 451 & 56717 & 9695 & 0 & 27 & 5 & Clusters of cases \\
\hline China & 210 & 90351 & 61 & 11 & 4728 & 3 & Clusters of cases \\
\hline Papua New Guinea & 98 & 459 & 51 & 1 & 5 & 1 & Community transmission \\
\hline Malaysia & 60 & 9317 & 288 & 0 & 125 & 4 & Clusters of cases \\
\hline New Zealand & 54 & 1378 & 286 & 0 & 22 & 5 & Clusters of cases \\
\hline Viet Nam & 26 & 1040 & 11 & 6 & 32 & $<1$ & Clusters of cases \\
\hline Mongolia & 3 & 301 & 92 & 0 & 0 & $<1$ & Sporadic cases \\
\hline Brunei Darussalam & 1 & 144 & 329 & 0 & 3 & 7 & Sporadic cases \\
\hline Cambodia & 0 & 273 & 16 & 0 & 0 & $<1$ & Sporadic cases \\
\hline Fiji & 0 & 28 & 31 & 1 & 2 & 2 & Sporadic cases \\
\hline Lao People'S Democratic Republic & 0 & 22 & 3 & 0 & 0 & $<1$ & Sporadic cases \\
\hline \multicolumn{8}{|l|}{ Territoriesii } \\
\hline Guam & 527 & 1339 & 7934 & 3 & 10 & 59 & Clusters of cases \\
\hline French Polynesia & 184 & 482 & 1716 & 0 & 0 & $<1$ & Sporadic cases \\
\hline $\begin{array}{l}\text { Northern Mariana Islands } \\
\text { (Commonwealth of The) }\end{array}$ & 2 & 56 & 973 & 0 & 2 & 35 & Pending \\
\hline New Caledonia & 0 & 23 & 81 & 0 & 0 & $<1$ & Sporadic cases \\
\hline Subtotal for all regions & 1782811 & 24853399 & - & 38088 & 838911 & - & \\
\hline${ }^{\dagger}$ Other & 0 & 741 & - & 0 & 13 & - & Not applicable \\
\hline Grand total & 1782811 & 24854140 & - & 38088 & 838924 & - & \\
\hline
\end{tabular}

See data, table and figure notes 


\section{Key weekly updates}

- WHO has issued updated interim guidance on hotels and other accommodation facilities to help the sector protect the safety of staff and clients. See also the Q\&A's on Staying at hotels and accommodation establishments and COVID-19 and Working in hotels and COVID-19.

- WHO has also released guidance for shipowners, seafarers, unions and associations and associated authorities for promoting public health measures on cargo ships and fishing vessels. This complements guidance for employers to make sure they implement containment measures at workplaces and the related $\underline{Q \& A}$.

- Speaking at his regular media briefing on 27 August, WHO Director-General Dr Tedros highlighted the momentous achievement of eradicating wild poliovirus in Africa and sleeping sickness in Togo. Dr Tedros went on to say that "globally, we need the same spirit of solidarity and partnership that are helping to end polio and sleeping sickness to end the COVID-19 pandemic."

- The results of a WHO survey conducted to assess the impact of the COVID-19 pandemic on up to 25 essential health services in countries show disruptions of essential health services in nearly all countries, and more so in lower-income than higher-income countries. The great majority of service disruptions were partial, which was defined as a change of $5-50 \%$ in service provision or use.

- WHO has published an overview of the structure, methodology, and assumptions used by the COVID-19 Essential Supplies Forecasting Tool (ESFT). The ESFT is designed to help governments, partners, and other stakeholders to estimate potential requirements for essential supplies to respond to the current pandemic of COVID-19. See also the ESFT Frequently Asked Questions. 


\section{Technical guidance and other resources}

- Technical guidance

- WHO Coronavirus Disease (COVID-19) Dashboard

- Weekly COVID-19 Operations Updates

- WHO COVID-19 Case definition

- COVID-19 Supply Chain Inter-Agency Coordination Cell Weekly Situational Update

- Updates from WHO regions

- African Region

- Eastern Mediterranean Region

- European Region

- Research and Development

- Online courses on COVID-19 and in additional national languages

- The Strategic Preparedness and Response Plan (SPRP) outlining the support the international community can provide to all countries to prepare and respond to the virus

\section{Recommendations and advice for the public}

- Protect yourself

- Questions and answers

- Travel advice

- EPI-WIN: tailored information for individuals, organizations and communities

\section{Data, table and figure notes}

Data presented is based on official laboratory-confirmed COVID-19 case and deaths reported to WHO by country/territories/areas, largely based upon WHO case definitions and surveillance guidance. While steps are taken to ensure accuracy and reliability, all data are subject to continuous verification and change, and caution must be taken when interpreting these data as several factors influence the counts presented, with variable underestimation of true case and death incidence, and variable delays to reflecting these data at global level. Case detection, inclusion critiera, testing strategies, reporting practice, and data cut-off and lag times differ between countries/territories/areas. A small number of countries/territories/ areas report combined probable and laboratory-confirmed cases; efforts are underway to identify these for notation in the data table. Differences are to be expected between information products published by WHO, national public health authorities, and other sources.

The designations employed, and the presentation of these materials do not imply the expression of any opinion whatsoever on the part of WHO concerning the legal status of any country, territory or area or of its authorities, or concerning the delimitation of its frontiers or boundaries. Dotted and dashed lines on maps represent approximate border lines for which there may not yet be full agreement. Countries, territories and areas are arranged under the administering WHO region. 
The mention of specific companies or of certain manufacturers' products does not imply that they are endorsed or recommended by WHO in preference to others of a similar nature that are not mentioned. Errors and omissions excepted, the names of proprietary products are distinguished by initial capital letters.

${ }^{[1]}$ All references to Kosovo should be understood to be in the context of the United Nations Security Council resolution 1244 (1999). In the map, number of cases of Serbia and Kosovo (UNSCR 1244, 1999) have been aggregated for visualization purposes.

i Transmission classification is based on a process of country/territory/area self-reporting. Classifications are reviewed on a weekly basis and may be revised as new information becomes available. Differing degrees of transmission may be present within countries/territories/areas; classification is based on the highest category reported within a country/territory/area. Categories:

- No cases: with no confirmed cases;

- Sporadic cases: with one or more cases, imported or locally detected;

- Clusters of cases: experiencing cases, clustered in time, geographic location and/or by common exposures;

- Community transmission: experiencing larger outbreaks of local transmission defined through an assessment of factors including, but not limited to: large numbers of cases not linkable to transmission chains; large numbers of cases from sentinel laboratory surveillance; and/or multiple unrelated clusters in several areas of the country/territory/area;

- Pending: transmission classification has not been reported to WHO.

ii "Territories" include territories, areas, overseas dependencies and other jurisdictions of similar status.

${ }^{+}$Other: includes cases reported from international conveyances.

\section{Country, territory, or area-specific notes, updates and errata}

Due to public health authorities conducting data reconciliation exercises which remove large numbers of cases or deaths from their total counts, negative numbers may be displayed in the new cases/deaths columns as appropriate. When additional details become available that allow the subtractions to be suitably apportioned to previous days, graphics will be updated accordingly. See the log of major changes and errata for details. Prior situation reports will not be edited; see covid19. who.int for the most up-todate data. 